\title{
AUTHOR INDEX \\ Volume 5, 1995
}

Agoudov, N.V. \& Malakhov, A.N., On the effect of fluctuations on an intermittent laminar motion

Aguirre, L.A., see Billings

Aguirre, L.A. \& Billings, S.A., Retrieving dynamical invariants from chaotic data using NARMAX models

Aidun, C.K., see Poliashenko

Albrecht, K.-F., see Ebeling

Alexeyev, A.A. \& Shalfeev, V.D., Chaotic synchronization of mutually-coupled generators with frequency-controlled feedback loop

Almirantis, Y. \& Kaufman, M., Chiral selection of rotating waves in a reaction-diffusion system: The effect of a circularly polarized electromagnetic field

Alsedà, L., Guaschi, J., Los, J., Mañosas, F. \& Mumbrú, P., "Connect-the-dots" tree maps

Alvarez-Ramírez, J., Border-collision crises in a two-dimensional map

Anderson, A.R.A. \& Sleeman, B.D., Wave front propagation and its failure in coupled systems of discrete bistable cells modelled by Fitzhugh-Nagumo dynamics

Anishchenko, V.C., Vadivasova, T.E., Astakhov, V.V., Sosnovtseva, O.V., Wu, C.W. \& Chua, L.O., Dynamics of two coupled Chua's circuits

Anishchenko, V.C., Vadivasova, T.E., Postnov, D.E., Sosnovtseva, O.V., Wu, C.W. \& Chuo, L.O., Dynamics of the nonautonomous Chua's circuit

Ashwin, P. \& Swift, J.W., Torus doubling in four weakly coupled oscillators

Astakhov, V.V., see Anishchenko

Aston, P.J., Bifurcation and chaos in iterated maps with $O(2)$ symmetry

Aston, P.J. \& Bird, C.M., Analysis of the control of chaos-rate of convergence

Aston, P.J. \& Dellnitz, M., Symmetry breaking bifurcations of chaotic attractors

Atlee Jackson, E., OPCL migration controls between five attractors of the Chua system

Baker, B.M., see Kline

Baldwin, S., Toward a theory of forcing on maps of trees

Balibrea, F. \& Smítal, J., A characterization of the set $(f) \backslash(f)$ for continuous maps of the interval with zero topological entropy

Balibrea, F., Esquembre, F. \& Linero, A., Smooth triangular maps of type $2^{\infty}$ with positive topological entropy

Baranovsky, A. \& Daems, D., Design of one-dimensional chaotic maps with prescribed statistical properties

Barbé, A., Haeseler, F.V., Peitgen, H.-O. \& Skordev, G., CoarseGraining invariant patterns of one-dimensional two-state linear cellular automata

Barbé, A.M., On a class of fractal matrices. III. Limit structures and hierarchical iterated function systems

Barbi, M., see Petracchi

Barugola, A., Cathala, J.C. \& Mira, C., Extensions of the notion of chaotic area in second-order endomorphisms

Beck, C., see Gürbüz

Béda, P.B., The attitude stability of the stationary motions of a Dumbbell satellite in the case of air drag

Bedding, S. \& Briggs, K., Iteration of quaternion maps

Bellomo, P., The spectral statistics of triangular quantum billiards

Benedettini, F. \& Moon, F.C., Experimental dynamics of a hanging cable carrying two concentrated masses
Bernhardt, C., Self-Similarity maps for the set of unimodal cycles Billings, S.A., see Aguirre

Billings, S.A. \& Aguirre, L.A., Effects of the sampling time on the dynamics and identification of nonlinear models

Bird, C.M., see Aston

Bishop, S.R., see Galvanetto

Bishop, S.R., see Clifford

Bishop, S.R., see Xu

Block, L., Blokh, A.M. \& Coven, E.M., Zero entropy permutations Blokh, A.M., see Block

Briggs, K., see Bedding

Briseghella, L., see Galvanetto

Brøns, M., see Sturis

Bruin, H., Combinatorics of the kneading map

Bub, G. \& Glass, L., Bifurcations in a discontinuous circle map. A theory for a chaotic cardiac arrhythmia

Carcasses, J.-P., Singularities of the parametric plane of an $n$-dimensional map. Determination of different configurations of fold and flip bifurcation curves

63

Carcasses, J.-P., A necessary and sufficient condition to have $r$ pairs of complex multipliers with modulus equal to unity in the case of an n-dimensional map

Cathala, J.C., see Barugola

Celletti, A. \& Froeschlé, C., On the determination of the stochasticity threshold of invariant curves

Chakravarthy, V.S. \& Ottino, J.M., Mixing studies using horseshoes

Chas, M. \& Silberger, S., Open problems session

Chay, T.R., Bifurcations in heart rhythms

1677

Chay, T.R., Fan, Y.S. \& Lee, Y.S., Bursting, spiking, chaos, fractals and universality in biological rhythms

Chen, G., see Lai

Chen, P.Y., see Feeny

1157 Chillemi, S., see Petracchi

Chin, C. \& Nayfeh, A.H., Parametrically excited nonlinear twodegree-of-freedom systems with nonsemisimple one-to-one resonance

Chin, C., Nayfeh, A.H. \& Mook, D.T., The response of a nonlinear system with a nonsemisimple one-to-one resonance to a combination parametric resonance

Chua, L.O., see Muñuzuri

Chua, L.O., see Zheleznyak

Chua, L.O., see Pivka

Chua, L.O., see Murali

Chua, L.O., see Maistrenko

Chua, L.O., see de Castro

Chua, L.O., see $W u$

Chua, L.O., see Sharkovsky

Chua, L. O., see Anishchenko

Chua, L.O., see Anishchenko

1325

ifford, M.J. \& Bishop, S.R., Estimation of symmetry breaking and escape by observation of manifold tangencies $\quad 883$

Coven, E.M., see Block

1331

1585

D., see Baranovsky

Dames, C., see Pantazelou

de Castro, M., Pérez-Muñuzuri, V., Chua, L.O. \& Pérez-Villar, V., Complex feigenbaum's scenario in discretely-coupled arrays of nonlinear circuits

Dedieu, H., see Schweizer 
Dellnitz, M., see Aston

Dellnitz, M., Field, M., Golubitsky, M., Ma, J. \& Hohmann, A., Cycling chaos

Dellnitz, M., Golubitsky, M., Hohmann, A. \& Stewart, I., Spirals in scalar reaction-diffusion equations

Deng, B., Constructing Lorenz type attractors through singular perturbations

Deregel, Ph., see Sharkovsky

Dmitriev, A.S., Panas, A.I. \& Starkov, S.O., Experiments on speech and music signals transmission using chaos

Doerner, R., Hübinger, B. \& Martienssen, W., Adaptive orbit correction in chaos control

Douglass, J., see Pantazelou

Dumbell, K.D., see Small

Ebeling, W., Pösche1, T. \& Albrecht, K.-F., Entropy, transinformation and word distribution of informationcarrying sequences

Elbert, T., see Kowalik

Eleonsky, V.M. \& Korolev, V.G., On bifurcations in the Schrödinger problem admitting of an existence of nonlinear shift operators

Esquembre, F., see Balibrea

Fagella, N., Limiting dynamics for the complex standard family

Fan, Y.S., see Chay

Fedorenko, V., Simple and complex dynamics for one-dimensional manifold maps

Feeny, B.F., Moon, F.C., Chen, P.Y. \& Mukherjee, S., Chaotic mixing in rigid, perfectly plastic material

Feichtinger, G., Ghezzi, L.L. \& Piccardi, C., Chaotic behavior in an advertising diffusion model

Field, M., see Dellnitz

Fournier-Prunaret, D, see $U h l$

Freeman, W.J., see Kay

Froeschlé, C., see Celletti

Froyland, G., Judd, K., Mees, A.I., Watson, D. \& Murao, K., Constructing invariant measures from data

Galias, Z., New method for stabilization of unstable periodic orbits in chaotic systems

Galvanetto, U., Bishop, S.R. \& Briseghella, L., Mechanical stickslip vibrations

Ghezzi, L.L., see Feichtinger

Gilli, M., A Spectral approdch for chaos prediction in delayed cellular neural networks

Gillot, C. \& Llibre, J., Periods for maps of the figure-eight space

Glass, L., see $B u b$

Goldsztein, G. \& Strogatz, S.H., Stability of synchronization in networks of digital phase-locked loops

Golubitsky, M., see Dellnitz

Golubitsky, M., see Dellnitz

Gómez-Gesteira, $M$., see Muñuzuri

Gomez-Gesteira, M., see Mosquera

Gonchenko, S.V. \& Shil'nikov, L.P., On geometrical properties of two-dimensional diffeomorphisms with homoclinic tangencies

Gontar, V., see Gutman

González, G.A., Controlling chaos of an uncertain lozi system via adaptive techniques

Goswami, B.K., Observation of some new phenomena involving period tripling and period doubling

Govaerts, W., Bordered matrices and singularities of large nonlinear systems

Grimshaw, R.H.J., see Tian

Grosu, I., see Jackson

Grotta Ragazzo, C., Chaotic oscillations of a buckled beam

Guaschi, J., see Alseda
Guaschi, J. \& Llibre, J., Periodic points of $C^{1}$ maps and the asymptotic Lefschetz number

Gürbüz, G. \& Beck, C., Sunspot activity curves and intermittent chaotic behavior

Gutman, M. \& Gontar, V., Route to chaos via inverse cascade of continuous bifurcations

Haeseler, F.V., see Barbé

Hasler, M., see Schweizer

$\mathrm{He}, \mathrm{K}$., see $\mathrm{Hu}$

Hohmann, A., see Dellnitz

Hohmann, A., see Dellnitz

Hsu, C.S., Global analysis of dynamical systems using posets and digraphs

$\mathrm{Hu}, \mathrm{B}$., see Liu

Hu, G., Qu, Z. \& He, K., Feedback control of chaos in spatiotemporal systems

Hübinger, B., see Doerner

Jackson, E.A. \& Grosu, I., Toward experimental implementations of migration controls

Janková, K. \& Smítal, J., Maps with random perturbations are generically not chaotic

Jiménez López, V., Order and chaos for a class of piecewise linear maps

Joiner, K.M., see Mahaffy

Judd, K., see Froyland

Kappos, E., The conley index and global bifurcations. I. Concept and theory

Karytinos, A., see Papaioannou

Kaufman, M., see Almirantis

Kawachi, M., see Nara

Kay, L., Shimoide, K. \& Freeman, W.J., Comparison of EEG time series from rat olfactory system with model composed of nonlinear coupled oscillators

Kennedy, M.P., see Schweizer

Kennedy, M.P., A nonlinear dynamics interpretation of algorithmic $A / D$ conversion

Kline, R.P. \& Baker, B.M., A dynamical systems approach to membrane phenomena underlying cardiac arrhythmias

Koch, M., Tetzlaff, R. \& Wolf, D., Fluctuations in the state of chaos-chaos intermittency of Chua's circuit

Koltsova, O.Yu. \& Lerman, L.M., Periodic and homoclinic orbits in a two-parameter unfolding of a Hamiltonian system with a homoclinic orbit to a saddle-center

Kolyada, S. \& Snoha, L., On topological dynamics of sequences of continuous maps

Korolev, V.G., see Eleonsky

Kowalik, Z.J. \& Elbert, T., A practical method for the measurements of the chaoticity of electric brain and magnetic activity

Kroon, M. \& Stewart, I., Detecting the symmetry of attractors for six oscillators coupled in a ring

Kurths, J., see Rosenblum

Lai, D. \& Chen, G., Computing the distribution of the Lyapunov exponent from time series: The one-dimensional case study

Lakshmanan, M., see Murali

Lee, Y.S., see Chay

Lega, J., see Proctor

Leonov, G.A., Pendulum with positive and negative dry friction. Continuum of homoclinic orbits

Lerman, L.M., see Koltsova

Li, J., see Liu

Linero, A., see Balibrea

Liu, Z., Hu, B. \& Li, J., Bifurcation sets and distributions of limit cycles in a Hamiltonian system approaching the principal deformation of a Z4-field
1369

1611

297

901

1243

1487

1085

809

901

1175

1767 
Llibre, J., see Gillot

Llibre, J., see Guaschi

Llibre, J., Paraños, J. \& Rodriguez, J.A., On the extension of Sharkovskit's theorem to connected graphs with non-positive euler characteristic

Los, J., see Alsedà

Ma, J., see Dellnitz

Mahaffy, J.M., Joiner, K.M. \& Zak, P.J., A geometric analysis of stability regions for a linear differential equation with two delays

Maistrenko, V.L., see Maistrenko

Maistrenko, Yu.L., Maistrenko, V.L., Vikul, S.I. \& Chua, L.O., Bifurcations of attracting cycles from time-delayed Chua's circuit

Maksimov, A.G., see Velarde

Maksimov, A.G., Nekorkin, V.I. \& Rabinovich, M.I., Soliton trains and $I-V$ characteristics of long Josephson junctions

Malakhov, A.N., see Agoudov

Mañosas, F., see Alsedà

Mansilla, R. \& Mateo-Reig, R., On the mathematical modelling of intronic sectors of the DNA molecule

Martienssen, W., see Doerner

Mateo-Reig, R., see Mansilla

Mees, A.I., see Paskota

Mees, A.I., see Paskota

Mees, A.I., see Froyland

Mira, C., see Barugola

Mira, C. \& Rauzy, C., Fractal aggregation of basin islands in twodimensional quadratic noninvertible maps

Misiurewicz, M., Thirty years after Sharkovskit's theorem

Mook, D.T., see Chin

Moon, F.C., see Feeny

Moon, F.C., see Benedettini

Mosquera, J., Gómez-Gesteira, M., Pérez-Muñuzuri, V., Muñuzuri, A.P. \& Pérez-Villar, V., Electric field influence on traveling wave propagation and stationary pattern formation

Moss, F., see Petracchi

Moss, F., see Pantazelou

Mukherjee, S., see Feeny

Müller, K.-H., see Plesser

Mumbrú, P., see Alsedà

Muñuzuri, A.P., see Mosquera

Muñuzuri, A.P., Pérez-Muñuzuri, V., Gómez-Gesteira, M. \& Chua, L.O., Spatiotemporal structures in discretely-coupled arrays of nonlinear circuits: A review

Murali, K., Lakshmanan, M. \& Chua, L.O., Controlling and synchronization of chaos in the simplest dissipative nonautonomous circuit

Murao, K., see Froyland

Murray, J.D., see Zhu

Nara, S., Davis, P., Kawachi, M. \& Totsuji, H., Chaotic memory dynamics in a recurrent neural network with cycle memories embedded by pseudo-inverse method

Nayfeh, A.H., see Chin

Nayfeh, A.H., see Chin

Nekorkin, V.I., see Maksimov

Nekorkin, V.I., see Velarde

Nicolis, C., see Shil'nikov

Nicolis, G., see Shil'nikov

Nusse, H.E. \& Yorke, J.A., Border-collision bifurcations for piecewise smooth one-dimensional maps

O'Cairbre, F., O'Farrell, A.G. \& O'Reilly, A., Bistability, bifurcation and chaos in a laser system

O'Farrell, A.G., see O'Cairbre

O'Reilly, A., see O'Cairbre
Ottino, J.M., see Chakravarthy

519

Ottino, J.M., see Shinbrot

955

Panas, A.I., see Dmitriev

Pantazelou, E., see Petracchi

Pantazelou, E., Dames, C., Moss, F., Douglass, J. \& Wilkens, L., Temperature dependence and the role of internal noise in signal transduction efficiency of crayfish mechanoreceptors

Papaioannou, G. \& Karytinos, A., Nonlinear time series analysis of the stock exchange: The case of an emerging market

Paraños, J., see Llibre

Paskota, M., Mees, A.I. \& Teo, K.L., Directing orbits of chaotic dynamical systems

Paskota, M., Mees, A.I. \& Teo, K.L., Geometry of targeting of chaotic systems

Peitgen, H.-O., see Barbé

Pérez-Muñuzuri, V., see Muñuzuri

Pérez-Muñuzuri, V., see Mosquera

Pérez-Muñuzuri, V., see de Castro

Pérez-Villar, V., see Mosquera

Pérez-Villar, V., see de Castro

Petracchi, D., Barbi, M., Chillemi, S., Pantazelou, E., Pierson, D., Dames, C., Wilkens, L. \& Moss, F., A test for a biological signal encoded by noise

Phillipson, P.E. \& Schuster, P., Map dynamics of reproduction

Piccardi, C., see Feichtinger

Pierson, D., see Petracchi

Pivka, L., Zheleznyak, A.L., Wu, C.W. \& Chua, L.O., On the generation of scroll waves in a three-dimensional discrete active medium

Plesser, T. \& Müller, K.-H., Fourier analysis of the complex motion of spiral tips in excitable media

Poliashenko, M. \& Aidun, C.K., Computational dynamics of ordinary differential equations

Pöschel, T., see Ebeling

Postnov, D.E., see Anishchenko

Prigogine, I., Why irreversibility? The formulation of classical and quantum mechanics for nonintegrable systems

Proctor, M.R.E. \& Lega, J., Secondary bifurcations and symmetry breaking as a route towards spatiotemporal disorder

Qu, Z., see $\mathrm{Hu}$

Rabinovich, M.I., see Maksimov

Rai, V., Modelling ecological systems

Rauzy, C., see Mira

Rodriguez, J.A., see Llibre

Romanenko, E.Yu., Sharkovsky, A.N. \& Vereikina, M.B., Selfstructuring and self-similarity in boundary value problems

Rosenblum, M. \& Kurths, J., A simple test for hidden periodicity in time series data

Schreiber, T., Efficient neighbor searching in nonlinear time series analysis

Schuster, P., see Phillipson

Schweizer, J., Kennedy, M.P., Hasler, M. \& Dedieu, H., Synchronization theorem for a chaotic system

Shalfeev, V.D., see Alexeyev

Sharkovskir., A.N., Coexistence of cycles of a continuous map of the line into itself

Sharkovsky, A.N., see Romanenko

Sharkovsky, A.N., Universal phenomena in some infinitedimensional dynamical systems

Sharkovsky, A.N., Deregel, Ph. \& Chua, L.O., Dry turbulence and period-adding phenomena from a I-D map with time delay

Shil'nikov, A., Nicolis, G. \& Nicolis, C., Bifurcation and predictability analysis of a low-order atmospheric circulation model

Shil'nikov, L.P., see Gonchenko 
Shimoide, K., see Kay

Shinbrot, T. \& Ottino, J.M., Maps, PDE's and solitary waves

Silberger, S., see Chas

Skordev, G., see Barbé

Sleeman, B.D., see Anderson

Small, A.J., Dumbell, K.D. \& Smith, P.D., Chaos in the Tang-

Mees-Chua model of threshold synchronisation

Smítal, J., see Janková

Smítal, J., see Balibrea

Smith, P.D., see Small

Snoha, L., see Kolyada

Sorger, G., Chaotic Ramsey equilibrium

Sosnovtseva, O.V., see Anishchenko

Sosnovtseva, O.V., see Anishchenko

Starkov, S.O., see Dmitriev

Stewart, I., see Kroon

Stewart, I., see Dellnitz

Strogatz, S.H., see Goldsztein

Struchkov, I.N., Permanent transient chaos in a two-dimensional non-invertible map

Sturis, J. \& Brøns, M., Chaos and chaotic transients in a forced model of the economic long wave: The role of homoclinic bifurcation to infinity

Swift, J.W., see Ashwin

Takens, F., Reduction entropy

Teo, K.L., see Paskota

Teo, K.L., see Paskota

Tetzlaff, R., see Koch

Tian, X. \& Grimshaw, R.H.J., Low-dimensional chaos in a perturbed Korteweg-de Vries equation

Totsuji, H., see Nara

Uhl, C., Fournier-Prunaret, D., Chaotic phenomena in an order 1 DPCM transmission system
Vadivasova, T.E., see Anishchenko $\quad 1525$

Vadivasova, T.E., see Anishchenko

1677

Velarde, M.G., Nekorkin, V.I. \& Maksimov, A.G., Further results on the evolution of solitary waves and their bound states of a dissipative Korteweg - De vries equation 831

Vereikina, M.B., see Romanenko $\quad 1407$

Vikul, S.I., see Maistrenko

Walsh, J.A., Rotation vectors for toral maps and flows: A tutorial 321

Watson, D., see Froyland $\quad 1181$

Wilkens, L., see Petracchi

Wilkens, L., see Pantazelou 101

Wolf, D., see Koch 271

Wu, C.W., see Pivka 313

Wu, C.W., see Anishchenko 1525

Wu, C.W., see Anishchenko 1677

Wu, C.W. \& Chua, L.O., Finding parameters for Chua's oscillator which is topologically conjugate to systems with a scalar nonlinearity

$\mathrm{Xu}, \mathrm{D}$. \& Bishop, S.R., A contraction-mapping-based control approach to stabilize chaotic systems

Yorke, J.A., see Nusse

Yu, X.H., Discretization chaos in the switching control system with finite switching values

Zak, P.J., see Mahaffy

Zheleznyak, A.L., see Pivka

Zheleznyak, A.L. \& Chua, L.O., Estimating the dimensional characteristics of two-dimensional patterns

Zhu, M. \& Murray, J.D., Parameter domains for generating spatial pattern: A comparison of reaction-diffusion and cellchemotaxis models
1741
1221

1205

1033 